\title{
Explicit design of \\ Time-varying Stabilizing Control Laws for \\ a Class of Controllable Systems without Drift
}

Jean-Baptiste Pomet

May 23, 1991, revised September 13, 1991

To appear in : Systems \& Control Letters (1992)

\begin{abstract}
This paper gives a systematic way to design time-variant feedback control laws for a class of controllable non-linear systems. This class contains a lot of systems which cannot be stabilized via a time-invariant feedback control law.

The interest of this work lies in the design method since a general existence result is already available. The techniques employed here are basic: they mainly involve classical Lyapunov analysis.
\end{abstract}

KEYWORDS : Nonlinear systems, Asymptotic stabilization, Controllability, Time-varying feedback.

This work was done while the author was visiting at INRIA Sophia-Antipolis

2004 route des lucioles

06565 Valbonne cédex (France)

His present address is : Laboratoire d'Automatique de Nantes, U.R.A. C.N.R.S. 823

E. C. N.

1, rue de la Noë

44072 NANTES cédex (France)

pomet@lan01.ensm-nantes.fr 


\section{Introduction}

Let us consider a control system of the form

$$
\dot{x}=\sum_{k=1}^{m} u_{k} f_{k}
$$

where $x$ lives in $\mathbb{R}^{n}$ and the controls $u_{k}$ are real. The $f_{k}$ 's are smooth vector fields.

Such affine control systems enjoy nice open-loop properties due to their symmetry (any trajectory can be followed in either direction by changing the sign of the controls). For instance, full rank of the Control Lie Algebra (i.e. the vector space spanned at any point by all the Lie brackets of the vector fields $f_{k}$ having dimension $n$ ) is sufficient for controllability in any usual sense. It has however been known for a long time that controllable nonlinear systems may fail to be stabilizable by time-invariant static or dynamic feedback. The link between controllability and stabilizability, or between open-loop and closed-loop properties in general is a topic of ongoing research, and actually one of the most challenging problems in nonlinear systems. For a survey on nonlinear stabilization, see for instance reference [12] by E. Sontag.

As a matter of fact, one can appreciate the gap between stabilizability and controllability for a system like (1) in the following (negative) result, which is a simple consequence of a necessary condition for feedback stabilization given by R. Brockett in [1]. Note that condition (2) does not contradict controllability, on the countrary. It is satisfied by our examples (30) and (36).

Proposition 0 If $m<n$ and

$$
\operatorname{Rank}\left\{f_{1}(0), \ldots, f_{m}(0)\right\}=m
$$

then there exists no continuous feedback law $u_{1}=u_{1}(x), \ldots, u_{m}=u_{m}(x)$ making 0 a locally asymptotically stable equilibrium point of the closed-loop system. There does not exist either any continuous dynamic feedback law $u_{1}=u_{1}(\xi, x), \ldots, u_{m}=u_{m}(\xi, x), \dot{\xi}=g(\xi, x)$ making $(\xi, x)=(0,0)$ a locally asymptotically stable equilibrium point of the closed-loop system.

Proof : Let $e_{o}$ be a nonzero vector linearly independent from $f_{1}(0), \ldots, f_{m}(0)$. By continuity, there is an $\epsilon>0$ such that for all $\left(x, u_{1}, \ldots, u_{m}\right)$ with $\|x\|<\epsilon$, the vector $\sum_{k=1}^{m} u_{k} f_{k}(x)$ is different from $\lambda e_{o}$ for any $\lambda$ in $\mathbb{R}$. Therefore, the map $\left(x, u_{1}, \ldots, u_{m}\right) \mapsto \sum_{k=1}^{m} u_{k} f_{k}(x)$ does not map the neighborhood $[-\epsilon, \epsilon]^{n+m}$ of 0 in $\mathbb{R}^{n+m}$ into a neighborhood of 0 in $\mathbb{R}^{n}$. This proves the proposition because the necessary condition for local asymptotic stabilizability of (1) given in [1] is violated. This condition is also necessary for dynamic feedback stabilization.

This impossibility to stabilize by means of a state feedback has been noticed on many occasions in the area of mechanical systems with nonholomic constraints; see, for instance, the works of C. Samson [9] and G. Campion, B. d'Andréa-Novel and G. Bastin [2]. With this motivation, C. Samson tried, successfully, time varying control laws to stabilize some nonholonomic robots, see $[9,10]$. This showed the power of this class of control, and is the starting point of some research on time-varying stabilization for nonlinear systems. It has 
recently been proved by J.-M. Coron in [3] that system (1) is stabilizable by means of a periodic time-varying feedback if its Control Lie Algebra has rank $n$ except possibly at the origin, but the existence proof does not provide explicitely the control law. R. Sépulchre, using a different approach, also gives some time-varying control laws in some other situations, see $[11]$.

The present paper presents a constructive approach allowing to derive an explicit control law and Lyapunov function under an assumption which is more restrictive than full rank Lie Algebra. By explicit, we do not necessarily mean that the control law is an algebraic expression of some functions fixed by the designer and the components of the vector fields, although this is the case in section 2.2 : in the more general situation considered here (section 2.1 ), writing the control laws involves the flow of an ordinary differential equation. Note that this exhibits almost the same degree of complexity as performing feedback linearization for a feedback linearizable system (i.e. in particular finding the linearizing coordinates), which involves the flow of several ordinary differential equations, see $[7,5]$.

The assumption we make is the following: we require the Control Lie Algebra to have full rank and to be generated by iterated Lie Brackets involving at most one $f_{k}$ different from $f_{1}$, and only once :

Assumption 1 For any $x \neq 0$,

$$
\begin{array}{rlll}
\operatorname{Rank}\left\{f_{1}(x),\right. & f_{2}(x), & \ldots & f_{m}(x) \\
& {\left[f_{1}, f_{2}\right](x),} & \ldots & {\left[f_{1}, f_{m}\right](x), \ldots} \\
\ldots & \operatorname{ad}_{f_{1}}^{j} f_{2}(x), & \ldots & \left.\operatorname{ad}_{f_{1}}^{j} f_{m}(x), \ldots \ldots\right\}=n
\end{array}
$$

Remark 1 : Of course, $f_{1}$ does not play a privileged role. If there is a linear combination of the $f_{k}$ 's such that the $f_{k}$ 's and all the iterated Lie brackets of this linear combination with them span $\mathbb{R}^{n}$, then one may satisfy assumption 1 by making a preliminary feedback transformation which "renames" this linear combination $f_{1}$. We take advantage of this possibility in the treatment of the 4-dimensional example (36) in section 3.2.

It is actually possible to extend the method presented here from the case of assumption 1 to the more general case of full rank Control Lie Algebra for $x \neq 0$. This extension makes use of results contained in [3], it is presented in the future publication [4] and it is briefly outlined in section 3.3 .

The techniques employed here are based on Lyapunov analysis and LaSalle's invariance principle [8]. They are similar to these employed by Jurjevic and Quinn in [6], see remark 5 in the proof of theorem 1 (section 4.2).

The paper is organized as follows : in section 2, a class of time-varying stabilizing control laws for system (1) is proposed, section 3 illustrates the method by applying it to two examples from non-holonomic robots, and outlines a possible extension, section 4 gives a proof of the stability results stated in section 2 .

\section{The control laws and the main results}

Here, we explicitly build a family of control laws, with a degree of freedom represented by a function $\alpha$. Section 2.1 gives a general way of building a time-varying stabilizing control 
law under assumption 1. This method involves solving a linear PDE, i.e. the expression of the control law (and the Lyapunov function) involves the flow of a certain ordinary differential equation. Section 2.2 gives, under the additional assumption 2, a way to derive some stabilizing control laws without solving any differential relations, except possibly for finding some coordinates in which this assumption is met : the control laws are actually algebraic functions of the components of the vector fields in these coordinates and of some variables and functions to be chosen by the designer.

\subsection{A general expression under assumption 1}

The following proposition gives a periodic time-varying function, used in the design of the control laws, and meant to be a Lyapunov function for the final closed-loop system.

For a given time-varying function $\alpha(t, x)$, we may consider the time-varying ordinary differential equation

$$
\dot{x}=\alpha(t, x) f_{1}(x),
$$

and define its "general" solution $\psi\left(t_{1}, t_{2}, x\right)$ : it is the value at time $t_{1}$ of the solution of (4) which started from $x$ at time $t_{2}$. In other words,

$$
\begin{aligned}
\frac{\partial \psi}{\partial t_{1}}\left(t_{1}, t_{2}, x\right) & =\alpha\left(t_{1}, \psi\left(t_{1}, t_{2}, x\right)\right) f_{1}\left(\psi\left(t_{1}, t_{2}, x\right)\right) \\
\psi(t, t, x) & =x .
\end{aligned}
$$

$\psi$ is defined, from classical results on ordinary differential equations, on an open subset of $\mathbb{R} \times \mathbb{R} \times \mathbb{R}^{n}$ containing the points $\left(t_{1}, t_{2}, x\right)$ such that $t_{1}=t_{2}$.

Proposition 1 Let $\alpha$ be a time-varying function (i.e. a function from $\mathbb{R} \times \mathbb{R}^{n}$ to $\mathbb{R}$ ), and let us suppose that:

- it is $2 \pi$-periodic and "odd" with respect to time, and vanishes for $x=0$ :

$$
\begin{aligned}
\alpha(t+2 \pi, x) & =\alpha(t, x) \quad \forall(t, x) \\
\alpha(-t, x) & =-\alpha(t, x) \quad \forall(t, x) \\
\alpha(t, 0) & =0 \quad \forall t
\end{aligned}
$$

- it satisfies the following majoration, for a certain $K>0$ :

$$
|\alpha(t, x)|\left\|f_{1}(x)\right\| \leq K(1+\|x\|) \quad \forall(t, x) .
$$

Then, the function $V$ given by

$$
V(t, x)=\frac{1}{2}\|\psi(0, t, x)\|^{2},
$$

where $\psi$ is given by (5), is well defined from $\mathbb{R} \times \mathbb{R}^{n}$ to $[0,+\infty)$ and enjoys the following properties :

- It is $2 \pi$-periodic with respect to time :

$$
V(t+2 \pi, x)=V(t, x)
$$


- $V$, as well as its derivative with respect to $x$, vanish if and only if $x=0$ :

$$
\begin{aligned}
V(t, x) & =0 \quad \Leftrightarrow \quad x=0 \\
\frac{\partial V}{\partial x}(t, x) & =0 \quad \Leftrightarrow \quad x=0
\end{aligned}
$$

- It is (the only) solution of the following linear PDE with initial conditions at $t=0$ :

$$
\begin{gathered}
\frac{\partial V}{\partial t}(t, x)+\alpha(t, x) \frac{\partial V}{\partial x}(t, x) f_{1}(x)=0 \\
V(0, x)=\frac{1}{2}\|x\|^{2}
\end{gathered}
$$

- For any positive real number $K$, the set

$$
\{x, V(t, x) \leq K \text { for some } t \in \mathbb{R}\} \text { is bounded } .
$$

Remark 2 : - In the expression of $V(10)$, the squared norm might be replaced by any proper positive function, vanishing only at 0 , and with no other singular point.

- (9) might be replaced by any condition ensuring that all the solutions of $\dot{x}=\alpha(t, x) f_{1}(x)$ are defined for any time.

- (7) might be replaced by $\alpha(T-t, x)=\alpha(t, x)$ for a certain $T \in \mathbb{R}$, or by any condition ensuring that all the solutions of $\dot{x}=\alpha(t, x) f_{1}(x)$ are $2 \pi$-periodic.

This proposition will be proved in section 4. It turns out that, for any $\alpha$ meeting (6), (7), (8), (9) the $2 \pi$-periodic time-varying function $V$ given by (10), may be assigned to be non-increasing along the closed-loop system by using the following $2 \pi$-periodic time-varying control law :

$$
\begin{aligned}
u_{1}(t, x) & =\alpha(t, x)-L_{f_{1}} V(t, x) \\
u_{2}(t, x) & =-L_{f_{2}} V(t, x) \\
& \vdots \\
u_{m}(t, x) & =-L_{f_{m}} V(t, x) .
\end{aligned}
$$

Under assumption 1 and a further requirement on $\alpha$, this control law asymptotically stabilizes (1). An explicit $\alpha$ meeting all the requirements may very easily be given, but we keep some freedom on $\alpha$ in order to be able, on some examples, to derive the simplest control laws we can. This is made precise by the following theorem :

Theorem 1 - Under assumption 1, and if $\alpha$ is chosen such that conditions (6), (7), (8), (9) of proposition 1 are satisfied and:

$$
\left.\begin{array}{r}
L_{f_{1}} V(t, x)=\ldots=L_{f_{m}} V(t, x)=0 \\
\alpha(t, x)=\frac{\partial \alpha}{\partial t}(t, x)=\frac{\partial^{2} \alpha}{\partial t^{2}}(t, x)=\ldots=0
\end{array}\right\} \Rightarrow x=0,
$$

then 0 is a globally uniformly asymptotically stable equilibrium point of the time-varying periodic closed-loop system obtained by applying the feedback law defined by (17), and the function $V$ is non-increasing along its solutions.

- It is always possible to find a function a meeting the required conditions. A possible choice $i s$

$$
\alpha(t, x)=\frac{\|x\|^{2}}{\left(1+\|x\|^{2}\right)\left(1+\left\|f_{1}(x)\right\|^{2}\right)} \sin t
$$


Remark 3 : A more general choice for $\alpha$ is

$$
\alpha(t, x)=\sin t \rho(x)
$$

where $\rho$ vanishes only for $x=0$ and is such that (9) is met. Many more choices are possible, but the expressions of $L_{F_{k}} V$ are needed, and $L_{F_{k}} V$ depends on $\alpha$. For example (36) (section 3.2 ), it is a priori possible not to include $\xi_{4}$ in the expression of $\alpha$ because, regardless of $\alpha$, $L_{f_{2}} V=\xi_{4}$ whenever $\alpha$ is zero.

\subsection{A simpler expression of the control laws in a less general case}

The above design of the control laws makes use of the function $V$ constructed in proposition 1. Its expression involves the flow of a differential equation. It is therefore very natural to look for some situations in which the expression of $V$ can be explicited without solving any such differential relation. It turns out that if the vector field $f_{1}$ is globally redressable, it is actually possible to give an explicit expression of $V$ and the control laws; it is the purpose of this section. As seen on example (36) in section 3.2, it may often be simpler, if possible, to make the necessary transformations (i.e. a preliminary static feedback and a change of coordinates) to meet assumption 2 than to use the general expressions given in previous section 2.1 .

Assumption 2 (simplifies the expression of the feedback law) There exists a system of coordinates $\left(x_{1}, \ldots, x_{n}\right)$ such that

$$
f_{1}=\frac{\partial}{\partial x_{1}} .
$$

Under this assumption, it is always possible to chose $V$ of the form

$$
V(t, x)=\frac{1}{2}\left(x_{1}+h\left(t, x_{2}, \ldots, x_{n}\right)\right)^{2}+\frac{1}{2} x_{2}^{2}+\ldots+\frac{1}{2} x_{n}^{2},
$$

with some requirements on $h$, so that, instead of taking $\alpha$ as a design parameter and solving the PDE (14)-(15) for $V$, we are now able to take $h$ as a design parameter and compute $\alpha$ so as to satisfy (14)-(15) :

Proposition 2 If assumption 2 is met, then for any function $h\left(t, x_{2}, \ldots, x_{n}\right)$ satisfying

$$
\begin{aligned}
h\left(t+2 \pi, x_{2}, \ldots, x_{n}\right) & =h\left(t, x_{2}, \ldots, x_{n}\right) \quad \forall(t, x) \\
h(t, 0) & =0 \quad \forall t,
\end{aligned}
$$

the function $V$ given by (22) and the function $\alpha$ given by

$$
\alpha(t, x)=-\frac{\partial h}{\partial t}\left(t, x_{2}, \ldots, x_{n}\right)
$$

are such that all the conclusions of proposition 1 (from (10) to (16)) are verified and in addition $\alpha$ satisfies (6) and (8). 
This proposition gives, from $h$, an $\alpha$ and a $V$, which are sufficient, as before, to construct the control law according to (17). We have the following theorem, which is a straighforward consequence of theorem 1 :

Theorem 2 - Under assumption 1 and 2, and if $h$ is chosen such that (23) and (24) are met and :

$$
\left.\begin{array}{r}
L_{f_{2}} W(x)=\ldots=L_{f_{m}} W(x)=0 \\
\frac{\partial h}{\partial t}(t, x)=\frac{\partial^{2} h}{\partial t^{2}}(t, x)=\frac{\partial^{3} h}{\partial t^{3}}(t, x)=\ldots=0
\end{array}\right\} \Rightarrow x=0,
$$

where

$$
W(x)=\frac{1}{2} x_{2}^{2}+\ldots+\frac{1}{2} x_{n}^{2},
$$

then 0 is a globally uniformly asymptotically stable equilibrium point of the time-varying periodic closed-loop system obtained by applying the feedback law defined by (17), with $\alpha$ and $V$ defined by (22) and (25). The function $V$ is non-increasing along its solutions.

- It is always possible to chose a function $h$ meeting the requirements. A possible choice is

$$
\begin{aligned}
& h(t, x)=\cos t\left(x_{2}^{2}+\ldots+x_{n}^{2}\right) \\
& \alpha(t, x)=\sin t\left(x_{2}^{2}+\ldots+x_{n}^{2}\right) .
\end{aligned}
$$

Remark 4 : Of course, some other choices than (28) are possible, as illustrated by the second choice for example (31) in section 3.1.

\section{Two examples}

\subsection{A three dimensional example.}

Let us consider the following 3-dimensional example :

$$
\begin{aligned}
& \dot{x_{1}}=u_{1} \\
& \dot{x_{2}}=x_{1} u_{2} \\
& \dot{x_{3}}=u_{2} .
\end{aligned}
$$

This is a reduced-order model for a three-wheeled cart with two independent rear motorized wheels. A time-varying feedback law has already been proposed for such a system in [9]. We show here that our method, and even the simplified one given in section 2.2, allows one to derive very simple feedback laws for this system. System (30) is of the form (1) with

$$
f_{1}=\frac{\partial}{\partial x_{1}} \quad ; \quad f_{2}=x_{1} \frac{\partial}{\partial x_{2}}+\frac{\partial}{\partial x_{3}} .
$$

Since $\left[f_{1}, f_{2}\right]=\frac{\partial}{\partial x_{2}}$, we have $: \operatorname{Rank}\left\{f_{1}, f_{2},\left[f_{1}, f_{2}\right]\right\}=3$, so that assumption 1 is satisfied. Assumption 2 is met as well in the original coordinates, so that we may follow the method of section 2.2 and take a $V$ of the form (22) :

$$
V(t, x)=\frac{1}{2}\left(x_{1}+h\left(t, x_{2}, x_{3}\right)\right)^{2}+\frac{1}{2} x_{2}^{2}+\frac{1}{2} x_{3}^{2} .
$$

Following (28), we may chose $h\left(t, x_{2}, x_{3}\right)=\left(x_{2}^{2}+x_{3}^{2}\right) \cos t$, and, from (17), the control law

$$
\begin{aligned}
& u_{1}=\left(x_{2}^{2}+x_{3}^{2}\right) \sin t-\left(x_{1}+\left(x_{2}^{2}+x_{3}^{2}\right) \cos t\right) \\
& u_{2}=-2\left(x_{1}+\left(x_{2}^{2}+x_{3}^{2}\right) \cos t\right)\left(x_{1} x_{2}+x_{3}\right) \cos t-\left(x_{1} x_{2}+x_{3}\right) .
\end{aligned}
$$


A simpler choice of $h$ is possible :

$$
h\left(t, x_{2}, x_{3}\right)=x_{2} \cos t
$$

also satisfies (26) since

$$
\begin{aligned}
L_{f_{1}} V & =x_{1}+x_{2} \cos t \\
L_{f_{2}} V & =\left(x_{1}+x_{2} \cos t\right) x_{1} \cos t+x_{2} x_{1}+x_{3} \\
\frac{\partial h}{\partial t} & =-x_{2} \sin t \\
\frac{\partial^{2} h}{\partial t^{2}} & =-x_{2} \cos t .
\end{aligned}
$$

With this choice of $h$, we find the following simpler control law :

$$
\begin{aligned}
& u_{1}=x_{2} \sin t-\left(x_{1}+x_{3} \cos t\right) \\
& u_{2}=-\left(x_{1}+x_{2} \cos t\right) x_{1} \cos t-\left(x_{1} x_{2}+x_{3}\right) .
\end{aligned}
$$

\subsection{A four dimensional example.}

The following system in $\mathbb{R}^{4}$ :

$$
\begin{aligned}
\dot{\xi}_{1}+i \dot{\xi}_{2} & =u_{1} \cos \xi_{4} e^{i \xi_{3}} \\
\dot{\xi}_{3} & =u_{1} \sin \xi_{4} \\
\dot{\xi}_{4} & =u_{2}
\end{aligned}
$$

is a reduced order model for a three-wheeled cart with a steering front wheel. $\xi_{1}$ and $\xi_{2}$ are the cartesian coordinates of the middle of the rear axle, $\xi_{3}$ is the angle between the axis of the cart and a fixed direction, and $\xi_{4}$ is the steering angle. A time-varying stabilizing feedback law has already been proposed for such a system in [10]. Let us show that :

- Our general method (section 2.1) applies. It is not easy however to write down the expression of the corresponding control law.

- Our simplified method (section 2.2) also applies, in some coordinates and after a preliminary static feedback transformation. We write down completely the corresponding control law.

- Our control law is different from the one proposed by C. Samson in [10], the expressions obtained in [10] are slightly simpler, and may be re-obtained through a generalization, outlined in section 3.3, of the method presented in section 2.1.

System (36) is of the form (1) :

$$
\dot{\xi}=u_{1} g_{1}(\xi)+u_{2} g_{2}(\xi)
$$

(we use $g$ instead of $f$ on purpose) with

$$
g_{1}=\cos \xi_{3} \cos \xi_{4} \frac{\partial}{\partial \xi_{1}}+\sin \xi_{3} \cos \xi_{4} \frac{\partial}{\partial \xi_{2}}+\sin \xi_{4} \frac{\partial}{\partial \xi_{3}} \quad g_{2}=\frac{\partial}{\partial \xi_{4}} .
$$

A simple computation gives:

$$
\begin{aligned}
& \operatorname{Rank}\left\{g_{1}, g_{2},\left[g_{1}, g_{2}\right],\left[g_{1},\left[g_{1}, g_{2}\right]\right]\right\}=4 \\
& {\left[g_{2},\left[g_{1}, g_{2}\right]\right]=g_{1}}
\end{aligned}
$$


so that assumption 1 is met with $f_{1}=g_{1}$ and $f_{2}=g_{2}$ but not with $f_{1}=g_{2}$ and $f_{2}=g_{1}$. Unfortunately assumption 2 is not met by $f_{1}=g_{1}$ since it may be proven that the vector field $g_{1}$ is not globally redressable. It is therefore not possible to use the method of section 2.2 with $f_{1}=g_{1}, f_{2}=g_{2}$. The method of section 2.1 does apply. The expressions of the Lyapunov function and the control law involve the flow of the time-variant ordinary differential equation

$$
\begin{aligned}
\dot{\xi}_{1}+i \dot{\xi}_{2} & =\alpha(t, \xi) \cos \xi_{4} e^{i \xi_{3}} \\
\dot{\xi}_{3} & =\alpha(t, \xi) \sin \xi_{4} \\
\dot{\xi}_{4} & =0
\end{aligned}
$$

with a choice of $\alpha$ such that (6), (8), (7), (9), and (18) are met. On this example, as on most examples, one may find a simpler $\alpha$ than the one given in (19). For example,

$$
\alpha(t, \xi)=\xi_{1} \sin t+\xi_{2} \sin 2 t+\xi_{3} \sin 3 t
$$

may easily be proved to meet conditions (6), (7), (8), (9), and (18) (the latter is met because $L_{f_{2}} V=\xi_{4}$ when $\left.\alpha(t, \xi)=0\right)$. However, even in this case, it is not easy to write explicitely the solutions of (40), and therefore the controls $u_{1}$ and $u_{2}$.

It turns out that it is simpler to perform a preliminary (static) feedback transformation in order to meet assumptions 1 and 2 together and apply section 2.2. If we define some new controls $v_{1}$ and $v_{2}$ by

$$
u_{1}=v_{2}+v_{1} \quad u_{2}=v_{1},
$$

system (36) reads

$$
\dot{\xi}=v_{1} f_{1}+v_{2} f_{2}
$$

with

$$
f_{1}=g_{2}+g_{1} \quad f_{2}=g_{1} .
$$

A very simple computation allows us to deduce from (39) that these $f_{1}$ and $f_{2}$ satisfy assumption 1. In addition, since the vector field $f_{1}$ has a constant nonzero component on $\frac{\partial}{\partial \xi_{4}}$, it meets assumption 2 , and as a matter of fact, the coordinates $x_{1}, x_{2}, x_{3}, x_{4}$ may be chosen as :

$$
\begin{aligned}
x_{1} & =\xi_{4} \\
x_{2} & =\xi_{3}+\cos \xi_{4}-1 \\
x_{3}+i x_{4} & =\xi_{1}+i \xi_{2}-e^{i\left(\xi_{3}+\cos \xi_{4}-1\right)} \int_{0}^{\xi_{4}} e^{-i \cos \tau} \cos \tau \mathrm{d} \tau
\end{aligned}
$$

Note that finding these coordinates involved the expression of the solutions of :

$$
\begin{aligned}
\dot{\xi}_{1}+i \dot{\xi}_{2} & =\cos \xi_{4} e^{i \xi_{3}} \\
\dot{\xi}_{3} & =\sin \xi_{4} \\
\dot{\xi}_{4} & =1
\end{aligned}
$$

whereas applying the method from section 2.1 involved the solutions of (40), which is more complicated due to the presence of $\alpha$. In coordinates $\left(x_{1} \ldots x_{4}\right)$, the vector fields are

$$
f_{1}=\frac{\partial}{\partial x_{1}} \quad f_{2}=\sin x_{1} \frac{\partial}{\partial x_{2}}+\chi_{3}\left(x_{1}, x_{2}\right) \frac{\partial}{\partial x_{3}}+\chi_{4}\left(x_{1}, x_{2}\right) \frac{\partial}{\partial x_{4}}
$$


with

$$
\chi_{3}\left(x_{1}, x_{2}\right)+i \chi_{4}\left(x_{1}, x_{2}\right)=e^{i x_{2}}\left(e^{-i \cos x_{1}} \cos x_{1}-i \sin x_{1} \int_{0}^{x_{1}} e^{-i \cos \tau} \cos \tau \mathrm{d} \tau\right) .
$$

The Lyapunov function $V$ is given by (22) with $h=\left(x_{2}^{2}+x_{3}^{2}+x_{4}^{2}\right) \cos t$ :

$$
V(t, x)=\frac{1}{2}\left(x_{1}+\left(x_{2}^{2}+x_{3}^{2}+x_{4}^{2}\right) \cos t\right)^{2}+\frac{1}{2} x_{2}^{2}+\frac{1}{2} x_{3}^{2}+\frac{1}{2} x_{4}^{2}
$$

and we get the following control law from (17) with $\alpha(t, x)=\left(x_{2}^{2}+x_{3}^{2}+x_{4}^{2}\right) \sin t$ :

$$
\begin{aligned}
u_{1}(t, x)= & \left(x_{2}^{2}+x_{3}^{2}+x_{4}^{2}\right) \sin t-x_{1}-\left(x_{2}^{2}+x_{3}^{2}+x_{4}^{2}\right) \cos t \\
u_{2}(t, x)=-2\left[\operatorname { c o s } t \left(x_{1}+\right.\right. & \left.\left.\left(x_{2}^{2}+x_{3}^{2}+x_{4}^{2}\right) \cos t\right)+1\right] \\
& \times\left[x_{2} \sin x_{1}+x_{3} \chi_{3}\left(x_{1}, x_{2}\right)+x_{4} \chi_{4}\left(x_{1}, x_{2}\right)\right] .
\end{aligned}
$$

In [10], C. Samson designed some different control laws for the same system, using

$$
z_{1}+i z_{2}=\left(\xi_{1}+i \xi_{2}\right) e^{-i \xi_{3}}
$$

instead of $\xi_{1}$ and $\xi_{2}\left(\left(z_{1}, z_{2}, \xi_{3}, \xi_{4}\right)\right.$ is $(x, y, \theta, \alpha)$ in [10]). We may rewrite his control laws as

$$
u_{j}=\alpha_{j}\left(t, z_{1}, z_{2}, \xi_{3}, \xi_{4}\right)-L_{g_{j}} V\left(t, z_{1}, z_{2}, \xi_{3}, \xi_{4}\right) \quad j=1,2
$$

where, $k$ being a function of $t, z_{2}, \xi_{3}$ ( $k$ is a degree of freedom),

$$
\begin{aligned}
V & =\frac{1}{2}\left(z_{1}+k\left(t, z_{2}, \xi_{3}\right)\right)^{2}+\frac{1}{2} z_{2}^{2}+\frac{1}{2} \xi_{3}^{2}+\frac{1}{2} \xi_{4}^{2}, \\
\alpha_{1} & =-\frac{\partial k}{\partial t}, \\
\alpha_{2} & =\left(z_{1} l-\frac{\sin \xi_{4}}{\xi_{4}} z_{1} z_{2}+\frac{\sin \xi_{4}}{\xi_{4}} \xi_{3}\right) \frac{\partial k}{\partial t}, \\
l\left(t, z_{1}, z_{2}, \xi_{3}, \xi_{4}\right) & =\frac{\sin \xi_{4}}{\xi_{4}}\left(z_{2}-\frac{\partial k}{\partial z_{2}} z_{1}+\frac{\partial k}{\partial \xi_{3}}\right)+\frac{\cos \xi_{4}-1}{\xi_{4}}
\end{aligned}
$$

( $l$ is $h$ in [10]). These laws are proved to yield stabilization for suitable choices of $k$, like $k\left(t, z_{2}, \xi_{3}\right)=z_{2} \sin t+\xi_{3} \cos t$.

From a computational point of view, both (52) and (50) give the controls as analytic fuctions of $t, \xi_{1}, \xi_{2}, \xi_{3}, \xi_{4}$ and involve some "unusual" analytic functions. It is however more convenient to have to compute $\frac{\sin \xi_{4}}{\xi_{4}}$ and $\frac{\cos \xi_{4}-1}{\xi_{4}}$ as in (52) than to have to compute $\int_{0}^{\xi_{4}} \cos (\cos \tau) \cos \tau \mathrm{d} \tau$ and $\int_{0}^{\xi_{4}} \sin (\cos \tau) \cos \tau \mathrm{d} \tau$, as in (50).

From a methodological point of view, the method leading to the controls in [9] is quite different from the one presented in the present paper, and (52) is only a way of re-writing the same controls. However, $V$ given by (53) may be checked to be the unique solution of

$$
\frac{\partial V}{\partial t}+\alpha_{1} L_{g_{1}} V+\alpha_{2} L_{g_{2}} V=0 \quad ; \quad V(0, \xi)=\frac{1}{2}\left(z_{1}+k\left(0, z_{2}, \xi_{3}\right)\right)^{2}+\frac{1}{2}\left(z_{2}^{2}+\xi_{3}^{2}+\xi_{4}^{2}\right) .
$$

It is therefore the function $V$ described in section 3.3 below, i.e. it satisfies (60)-(61) (changing the letter $f$ into $g$ ) and it is given by (10) and (59). Since (52) is exactly (62), the method of section 3.3 with $\alpha_{1}$ and $\alpha_{2}$ given by (54) does lead to the same control laws as 
[10]. It may be checked that (63) is met for instance if $k\left(t, z_{2}, \xi_{3}\right)=z_{2} \sin t+\xi_{3} \cos t$.

Note that : - Actually, to get $V$ exactly as in (53), another function than the squared norm has to be used in (10) if $k\left(0, z_{2}, \xi_{3}\right)$ is not zero (see also remark 2 ); for instance, if $k\left(t, z_{2}, \xi_{3}\right)=z_{2} \sin t+\xi_{3} \cos t$, then one has to chose in (10) the norm according to the matrix 1010

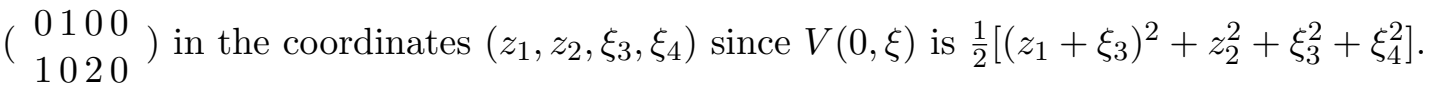
0001

- The expression of $V$ obtained from section 3.3 involves the solution $(\psi)$ of :

$$
\begin{aligned}
& \dot{z}_{1}=\alpha_{1}\left(\cos \xi_{4}+z_{2} \sin \xi_{4}\right) \\
& \dot{z}_{2}=-\alpha_{1} z_{1} \sin \xi_{4} \\
& \dot{\xi}_{3}=\alpha_{1} \sin \xi_{4} \\
& \dot{\xi}_{4}=\alpha_{2} .
\end{aligned}
$$

For $\alpha_{1}$ and $\alpha_{2}$ given by (54) and (55), and a general function $k\left(t, z_{2}, \xi_{3}\right)$, like $k\left(t, z_{2}, \xi_{3}\right)=$ $z_{2} \sin t+\xi_{3} \cos t$, the solutions of (58) cannot be completely written. Nevertheless, $V$ given by (10) has the explicit expression (58) because it turns out that taking the norm cancels out the parts of $\psi$ which cannot be simply expressed.

\subsection{An extension of the present method}

In the design proposed in section 2.1, one may use, instead of only one function $\alpha, m$ functions $\alpha_{1}, \ldots, \alpha_{m}$, each with the properties (6)-(9) ((9) becomes $\left|\alpha_{i}(t, x)\right|\left\|f_{i}(x)\right\| \leq K(1+$ $\|x\|)$ ), and define the Lyapunov function $V$ by (10) with $\psi$ the flow of $\dot{x}=\sum_{i=1}^{m} \alpha_{i}(t, x) f_{i}(x)$ instead of $\dot{x}=\alpha(t, x) f_{1}(x)$, i.e. with

$$
\begin{aligned}
\frac{\partial \psi}{\partial t_{1}}\left(t_{1}, t_{2}, x\right) & =\sum_{i=1}^{m} \alpha_{i}\left(t_{1}, \psi\left(t_{1}, t_{2}, x\right)\right) f_{i}\left(\psi\left(t_{1}, t_{2}, x\right)\right) \\
\psi(t, t, x) & =x
\end{aligned}
$$

instead of (5). Proposition 1 may easily be extended. Relation (14) is modified : defining, as in the proof of theorem 1 , a vector field $F_{o}$ on $\mathbb{R} \times \mathbb{R}^{n}$ (for the sake of clarity, we keep $\mathbb{R}$ instead of $S^{1}$ here and use $t$ instead of the "time modulo $2 \pi$ " $\theta$ ) by

$$
F_{0}=\frac{\partial}{\partial t}+\sum_{k=1}^{m} \alpha_{k} F_{k}
$$

with the $F_{k}$ 's defined as in the proof of theorem 1 (below equation (72)), (14) is replaced by :

$$
L_{F_{o}} V=0
$$

(which gives (14) if $\alpha_{1}=\alpha$ and $\alpha_{2}=\ldots=\alpha_{m}=0$ ). The control is then defined by :

$$
u_{k}=\alpha_{k}(t, x)-L_{F_{k}} V(t, x) \quad k=1 \ldots m .
$$

The same proof as for theorem 1 shows that, if the $\alpha_{i}$ 's have the above properties and :

$$
\left.\begin{array}{ll}
L_{a d_{F_{O}}^{j} F_{k}} V(t, x)=0 & \begin{array}{l}
k=1 \ldots m \\
j \geq 0
\end{array}
\end{array}\right\} \Rightarrow x=0
$$


then the control law (62) yields global asymptotic stability of the origin.

This extension is interesting for two different reasons :

- In the context of this paper (systems without drift meeting assumption 1), it gives some more degrees of freedom. As an aside consequence, it allows one to interpret the control laws proposed by C. Samson in [10] in the framework of this paper, see section 3.2.

- It may also extend to the more general case of controllable systems without drift, not necessarily meeting assumption 1 . This extension, using results from [3], is develloped and discussed in the forthcoming [4]. Let us briefly outline it. It is not easy to deduce from controllability (full rank Control Lie Algebra) an explicit expression of some $\alpha_{i}$ 's meeting (63), whereas assumption 1 allowed us to give the explicit expression (19) for $\alpha$. The central difficulty solved by J.-M. Coron in [3] is to establish existence of some $\alpha_{i}$ 's such that (his notations are not the same as here) :

$$
\operatorname{Rank}\left\{\operatorname{ad}_{F_{o}}^{j} F_{k}\left(\frac{3 \pi}{2}, x\right) \begin{array}{l}
k=1 \ldots m \\
j \geq 0
\end{array}\right\}=n \quad \forall x \neq 0
$$

From (13), this implies property (63), at least for $t=\frac{3 \pi}{2}$, and this is actually enough to conclude, via an extension of the proof of theorem 1.

\section{The proofs}

The proof of proposition $\mathbf{2}$ and theorem $\mathbf{2}$ are left to the reader. All the properties in proposition 2 can be checked by hand from (21), (22) and (25). Theorem 2 is actually a particular case of theorem 1 since it is easy to verify that $\alpha$ given by (25) satisfies all the properties required in theorem 1 except maybe (7) and (9) but these were only required to ensure that $V$ be well defined and periodic, which is here obvious from (22) and (23).

\subsection{Proof of proposition 1}

First of all, (9) implies that $\psi$, the "general solution" of (4) is defined for any $t_{1}, t_{2}$ and $x$ because the right-hand side of (4) sub-linear. $V$ is therefore defined all over $\mathbb{R} \times \mathbb{R}^{n}$.

To prove the other properties, let us explain where formula (10) comes from, and for which reasons it makes $V$ a solution of the PDE (14). This is just an application of the method of characteristics for solving the linear PDE (14) : (14) exactly means that the function $V$ is constant along the vector field $\frac{\partial}{\partial t}+\alpha f_{1}$ in $\mathbb{R} \times \mathbb{R}^{n}$. This vector field is complete from (9) and its flow at time $\tau$ is the diffeomorphism of $\mathbb{R} \times \mathbb{R}^{n}$ given by $(t, x) \mapsto \psi(t+\tau, t, x)$. Formula (10) therefore states that $V(t, x)$ is equal to $V(0, \xi)$ where $(0, \xi)$ is the image of $(t, x)$ under the flow of $\frac{\partial}{\partial t}+\alpha f_{1}$ at time $-t$, i.e. $(0, \xi)$ is the only point on the same integral curve of $\frac{\partial}{\partial t}+\alpha f_{1}$ as $(t, x)$ with a zero $t$-component. $V$ is therefore obviously constant on the integral curves of $\frac{\partial}{\partial t}+\alpha f_{1}$, and this proves (14). Note that (14) may also be checked by hand : by differentiating the identity

$$
\psi(\sigma, \tau, \psi(\tau, \sigma, \xi))=\xi
$$

with respect to $\tau$, one gets

$$
\frac{\partial \psi}{\partial t_{2}}(\sigma, \tau, \psi(\tau, \sigma, \xi))+\frac{\partial \psi}{\partial x}(\sigma, \tau, \psi(\tau, \sigma, \xi)) \frac{\partial \psi}{\partial t_{1}}(\tau, \sigma, \xi)=0 .
$$


From (5), one may, in (65), replace $\frac{\partial \psi}{\partial t_{1}}(\tau, \sigma, \xi)$ with $\alpha(\tau, \psi(\tau, \sigma, \xi)) f_{1}(\psi(\tau, \sigma, \xi))$. Applying the resulting identity for $\sigma=0, \tau=t$ and $\psi(\tau, \sigma, \xi)=x$ (i.e. $\xi=\psi(0, t, x)$ ), one gets

$$
\frac{\partial \psi}{\partial t_{2}}(0, t, x)+\frac{\partial \psi}{\partial x}(0, t, x) \alpha(t, x) f_{1}(x)=0 .
$$

Together with the expression (10) of $V$, this clearly implies that $V$ satisfies the PDE (14).

The fact that, for any $t_{1}$ and $t_{2}, \psi\left(t_{1}, t_{2}, 0\right)$ is zero (from (8)) and $x \mapsto \psi\left(t_{1}, t_{2}, x\right)$ is a diffeomorphism of $\mathbb{R}^{n}$ implies that $V$ defined by (10) satisfies (12), (13), (15) and (16) -(16) actually uses (11) established below.

The only remaining property to be proved is (11). Consider a solution $\gamma(t)$ of $(4) ;(7)$ implies that $\gamma(-t)=\gamma(t)$, and in particular that $\gamma(-\pi)=\gamma(\pi)$; this and (7) imply that $\gamma$ is $2 \pi$-periodic. $\psi$ is therefore $2 \pi$-periodic with respect to its first entry. Identity (64) applied twice, for $\sigma$ and $\sigma+2 \pi$, yields

$$
\psi(\sigma, \tau, \psi(\tau, \sigma, \xi))=\psi(\sigma+2 \pi, \tau, \psi(\tau, \sigma+2 \pi, \xi))=\xi
$$

and, since $\psi$ is $2 \pi$-periodic with respect to its first entry, $\psi(\sigma, \tau, \psi(\tau, \sigma, \xi))=\psi(\sigma, \tau, \psi(\tau, \sigma+$ $2 \pi, \xi)$ ), which implies $\psi(\tau, \sigma, \xi)=\psi(\tau, \sigma+2 \pi, \xi)$ because $x \mapsto \psi(\sigma, \tau, x)$ is one-to-one. $\psi$ is therefore $2 \pi$-periodic with respect to its second entry. This implies (11).

\subsection{Proof of theorem 1}

Remark 5: The stability proof given below is very much in the spirit of the one given in [6]. Actually, once the time-varying closed-loop system on $\mathbb{R}^{n}$ has been made a time invariant one on $S^{1} \times \mathbb{R}^{n}$, and once property (81) (lemma 1) has been established, one may notice that (81) is very similar to the "ad-condition" used in [6], and this allows to make almost the same application of LaSalle's invariance principle as in [6]. However, since it is somehow modified (one extra-dimension, invariant set $S^{1} \times\{0\}$ instead of equilibrium point, $\alpha$ vanishes...), we give the complete proof rather than refering the reader to [6].

The second point of the theorem is obvious : one may check by hand that $\alpha$ given by (19) meets all the requirements of the first point. Let us proceed with the proof of the first point.

Since the functions $\alpha, V, u_{1}, \ldots, u_{m}$ from $\mathbb{R} \times \mathbb{R}^{n}$ to $\mathbb{R}$ are $2 \pi$-periodic with respect to time, they induce naturally some functions from $S^{1} \times \mathbb{R}^{n}$ to $\mathbb{R}$, the circle $S^{1}$ being the quotient

$$
S^{1}=\mathbb{R} / 2 \pi \mathbb{Z} .
$$

The $2 \pi$-periodic time-variant closed-loop system can then be considered as a time-invariant system on $S^{1} \times \mathbb{R}^{n}$, given by

$$
\begin{aligned}
\dot{\theta} & =1 \\
\dot{x} & =\sum_{k=1}^{m} u_{k}(\theta, x) f_{k}(x)
\end{aligned}
$$

where $\theta$ lives in $S^{1}$. We may re-write (69), with $X=\left(\begin{array}{c}\theta \\ x\end{array}\right)$, into :

$$
\dot{X}=F(X)
$$


with

$$
F\left(\left(\begin{array}{c}
\theta \\
x
\end{array}\right)\right)=\left(\begin{array}{c}
1 \\
\sum_{k=1}^{m} u_{k}(\theta, x) f_{k}(x)
\end{array}\right)
$$

where the functions $u_{k}$ are given by (17). In other words

$$
F=\frac{\partial}{\partial \theta}+\sum_{k=1}^{m} u_{k} F_{k}
$$

where $F_{k}$ stands for the vector field on $S^{1} \times \mathbb{R}^{n}$ naturally induced by the original $f_{k}$ on $\mathbb{R}^{n}$ $\left(\dot{X}=F_{k}(X)\right.$ means $\left.\dot{\theta}=0, \dot{x}=f_{k}(x)\right)$.

The fact that $V$ decreases along the solutions of the closed-loop system is straightforward since the time-derivative of $V(x(t), \theta(t))$ along the solutions of the system is, from (69), (17) and (14),

$$
\dot{V}(\theta, x)=-\sum_{k=1}^{m}\left(L_{F_{k}} V(\theta, x)\right)^{2} .
$$

To prove that 0 is a unformly asymptotically stable equilibrium point of the time-variant closed-loop system, or equivalently that the invariant set $S^{1} \times\{0\}$ (or the periodic orbit $\dot{\theta}=1, x=0$ ) of system (69) is globally asymptotically attractive, we will use LaSalle's invariance principle [8].

Consider a solution of this closed loop system, with initial condition $\left(\theta_{o}, x_{o}\right)$. Since $V$ decreases along this solution, it stays in the set $V^{-1}\left(V\left(\theta_{o}, x_{o}\right)\right)$, which is compact from (16). Any solution is therefore bounded.

LaSalle's invariance principle states ([8] theorem 2) that, since $V$ decreases along any solution, any bounded solution converges to the largest invariant set included in

$$
A=\{(\theta, x), \dot{V}(\theta, x)=0\}=\left\{X \in S^{1} \times \mathbb{R}^{n}, L_{F_{k}} V(X)=0, k=1 \ldots m\right\}
$$

(where the second equality comes from (73)), which is also the reunion of all the integral curves of the vector field $F$ which stay in $A$.

From (17) and (74), we have :

$$
(\theta, x) \in A \Rightarrow\left\{\begin{aligned}
u_{1}(\theta, x) & =\alpha(\theta, x) \\
u_{2}(\theta, x) & =0 \\
& \vdots \\
u_{m}(\theta, x) & =0
\end{aligned}\right.
$$

so that the vector field $F$ coincides on $A$ with the vector field

$$
F_{o}=\frac{\partial}{\partial \theta}+\alpha F_{1}
$$

We therefore have to prove that the only integral curve of $F_{o}$ (i.e. solution of $\dot{X}=F_{o}(X)$ ) which stays in $A$ is $\dot{\theta}=1, x=0$.

From (74), the functions $L_{F_{k}} V$ are identically zero on these trajectories, and so are their derivatives : at any point $X=(\theta, x)$ of such a trajectory,

$$
\begin{array}{ll}
L_{F_{o}}^{j} L_{F_{k}} V(X)=0, & k=1, \ldots, m \\
& j \geq 0
\end{array}
$$


Now, from (14), $V$ is a first integral of $F_{o}$ :

$$
L_{F_{o}} V(X)=0 \quad \forall X \in S^{1} \times \mathbb{R}^{n},
$$

and it is not difficult to prove by induction on $k$ that this implies :

$$
L_{F_{o}}^{j} L_{F_{k}} V=L_{a d_{F_{o}}^{j} F_{k}} V .
$$

From (79) and (77), one can deduce that, at any point $(\theta, x)$ of a trajectory which stays in $A$,

$$
\begin{array}{ll}
L_{a d_{F_{o}}^{j} F_{k}} V(\theta, x)=0, & k=1, \ldots, m \\
& j \geq 0 .
\end{array}
$$

We shall now make use of following lemma :

Lemma 1 Under assumption 1, we have, at any $(\theta, x)$ such that $\alpha(\theta, x) \neq 0$,

$$
\operatorname{Rank}\left\{\operatorname{ad}_{F_{o}}^{j} F_{k}(\theta, x) \begin{array}{l}
k=1 \ldots m \\
j \geq 0
\end{array}\right\}=n .
$$

This lemma is proved further. To complete the proof of the theorem, let us first prove that $\alpha$ is identically zero on an integral curve of $F_{o}$ which stays in $A$. In order to prove this by contradiction, let us suppose that there is a point $(\theta, x)$ on such a curve where $\alpha(\theta, x) \neq 0$. From lemma 1 , the vectors $a d_{F_{o}}^{j} F_{k}(\theta, x), k=1, \ldots, m, j \geq 0$ span the subspace $\{0\} \times T_{x} \mathbb{R}^{n}$ of $T_{(\theta, x)}\left(S^{1} \times \mathbb{R}^{n}\right)$, and from (80) they are in the kernel of the linear form $d V(\theta, x)$; this implies that $d V$ is colinear to $d \theta$ at this point, which is impossible from (13).

This actually proves that, on an integral curve of the closed-loop system which stays in $A, F_{o}$ coincides with $\frac{\partial}{\partial \theta}$. Therefore, from (74) and the fact that $\alpha$ is identically zero on this trajectory, we have, at any point $(\theta, x)$ of this trajectory,

$$
\begin{array}{rll}
\frac{\partial^{j} \alpha}{\partial t^{j}}(\theta, x) & =0 & j \geq 0, \\
L_{f_{k}} V(\theta, x) & =0 & k=1, \ldots, m,
\end{array}
$$

which is equivalent to $x=0$ from (18). This proves that any solution of the closed-loop system which stays in $A$ statisfies $x=0$, and completes the proof from LaSalle's invariance principle.

Proof of lemma 1 : A simple inductive computation from (76) shows that

$$
\operatorname{ad}_{F_{o}}^{j} F_{k}=\alpha^{k} \operatorname{ad}_{F_{1}}^{j} F_{k}+\text { linear combination of } \operatorname{ad}_{F_{1}}^{j^{\prime}} F_{k^{\prime}} \quad \begin{array}{ll}
0 \leq j^{\prime} \leq j-1 \\
1 \leq k^{\prime} \leq m
\end{array}
$$

and this proves the lemma.

\section{Conclusion}

We have derived some stabilizing time-varying control laws, for a class of controllable nonlinear systems, characterized by assumption 1 . Existence has already been established independanlty in [3] in a more general situation. The interest of our approach is to provide the control laws themselves. Actually, it turns out that, for these systems with a zero drift vector field, there is a very wide range of possible stabilizing control laws. We have kept as many degrees of freedom as we possibly could without complicating too much the expressions. 


\section{Aknowledgements}

The author is very grateful to Claude Samson, from INRIA Sophia-Antipolis, France, for pointing out to him the power of time-varying feedback laws for stabilization, demonstrated on some mobile robots. The author wishes also to thank Laurent Baratchart, from INRIA Sophia-Antipolis, France, for some fruitful discussions on the subject, and Jean-Michel Coron, from Université Paris-Sud, Orsay, for suggesting condition (7) to ensure periodicity of all the solutions of (4).

\section{References}

[1] R. P. Brockett : Asymptotic stability and feedback stabilization, in: R.W. Brockett, R.S. Millman and H.H. Sussmann Eds., Differential Geometric Control Theory, Birkäuser Basel-Boston, 1983.

[2] G. Campion, B. d'Andréa-Novel and G. Bastin : Controllability and State-Feedback Stabilization of Non Holonomic Mechanical Systems, Int. Worshop in Adaptive and Nonlinear Control: Issues in Robotics, Grenoble, France, Springer-Verlag, 1990.

[3] J.-M. Coron : Global asymptotic stabilization for controllable systems without drift, preprint, 1991.

[4] J.-M. Coron and J.-B. Pomet : A remark on the design of time-varying control laws for controllable systems without drift, in preparation, 1991.

[5] L.R. Hunt, R. Su and G. Meyer : Design for multi-input nonlinear systems. in: R.W. Brockett, R.S. Millman and H.H. Sussmann Eds., Differential Geometric Control Theory, pp. 268-298, Birkäuser Basel-Boston, 1983.

[6] V. Jurdjevic and J.P. Quinn : Controllability and Stability. Journal of diff. eq. Vol. 28 (1978) pp. 381-389.

[7] B. Jakubczyk and B. Respondek : On linearization of control systems. Bull. Acad. Polonaise Sci. Ser. Sci. Math., 28 (1980) pp. 517-522.

[8] J.-P. LaSalle : Stability theory for ordinary differential equations. Journal of diff. eq. Vol. 4 (1968) pp. 57-65.

[9] C. Samson : Velocity and torque feedback control a nonholonomic cart. Int. Worshop in Adaptive and Nonlinear Control: Issues in Robotics, Grenoble, Springer, 1990.

[10] C. Samson : "Time-varying stabilization of a Nonholonomic Car-like Mobile Robot." Rapport INRIA Sophia-Antipolis, France, 1990.

[11] R. Sépulchre : private communication, 1991.

[12] E. D. Sontag : Feedback Stabilization of Nonlinear Systems, in : M.A. Kaashoek \& al. Eds., Robust Control of Linear Systems and Nonlinear Control, Proceedings of the Internationnal Symposium MTNS-89, Birkäuser Basel-Boston, 1990. 